\title{
Safety of perioperative dexamethasone administration in children: time for reflection?
}

\author{
Kevin Yee, MD • Robin G. Cox, MBBS
}

Received: 6 May 2013/Accepted: 7 June 2013/Published online: 19 June 2013

(c) Canadian Anesthesiologists' Society 2013

The first reported human therapeutic use of cortisone occurred in 1948. The patient was a 29-yr-old woman being treated at the Mayo Clinic for severe rheumatoid arthritis. After 60 days of treatment, her rheumatoid symptoms had improved, but treatment was terminated due to side effects such as hirsutism, acne, and hostile ideation. ${ }^{1}$ Thus, it was notable that this first trial of therapeutic corticosteroid therapy was terminated due to complications from the drug. The purpose of this editorial is to review the current evidence for the safety profile of dexamethasone, a synthetic corticosteroid, given that the drug is now widely used in the perioperative period in both children and adults. Concerns may linger in the minds of some anesthesiologists that potential side effects from even a single modest dose of dexamethasone might be important. Is there any evidence to support these fears? Are children at any more or less risk than adults for potential side effects? Is dose size and frequency an important consideration? Is there clear enough evidence to answer these questions in 2013, or is more research required?

Dexamethasone, a synthetic corticosteroid, is the product of research conducted by both the Schering Corporation and Merck Laboratories, as published in 1958. ${ }^{2}$ Over the following two decades, the use of dexamethasone as a potent anti-inflammatory agent was explored, and the drug was soon found to be beneficial in such clinical situations as third molar extraction ${ }^{3}$ and acute laryngotracheobronchitis (croup). ${ }^{4}$ The potent antiemetic

K. Yee, MD - R. G. Cox, MBBS ( $₫)$

Department of Anesthesia, Alberta Children's Hospital, University of Calgary, 2888 Shaganappi Trail N.W., Calgary, AB T3B 6A8, Canada

e-mail: robin.cox@albertahealthservices.ca action of dexamethasone was somewhat slower to be recognized, even though this had been shown in early studies. ${ }^{3}$ Dexamethasone found a role in the prevention of chemotherapy-induced vomiting in the $1980 \mathrm{~s},{ }^{5}$ but it took another decade or more before the implications for the control of postoperative nausea and vomiting (PONV) were fully appreciated. The Society for Ambulatory Anesthesia has critically evaluated the evidence for appropriate prevention and management of PONV, and the ensuing guidelines recommend dexamethasone as an effective prophylactic antiemetic in children at a dose of $150 \mu \mathrm{g} \cdot \mathrm{kg}^{-1}$, up to $5 \mathrm{mg}{ }^{6}$ Other perioperative effects of dexamethasone in children have focused on analgesia after procedures such as tonsillectomy ${ }^{7}$ and orchidopexy. ${ }^{8}$ The various benefits of dexamethasone are so well accepted that this drug is now used very widely in the perioperative period, usually as a single modest dose. Is there any evidence that this practice carries any significant risk particularly in pediatric patients?

The majority of evidence addressing harm from the use of corticosteroids comes from non-anesthetic literature. For example, corticosteroids are a mainstay in the treatment of asthma. In asthmatic children, daily oral or inhaled corticosteroids have been linked to decreased growth. ${ }^{9}$ Clinicians have also been concerned that corticosteroids may be associated with peptic ulceration, but the evidence suggests this to be more myth than reality. ${ }^{10,11}$ Corticosteroid-related psychological adverse effects are also possible in children, and they can manifest as aggressive behaviour and attention deficit hyperactivity disorder. This has been shown in studies on children undergoing long-term high-dose corticosteroid therapy for acute lymphoblastic leukemia and inflammatory bowel disease. ${ }^{12,13}$ Sleep disturbance has also been reported, but this has been shown to occur primarily in patients receiving 
high-dose steroid therapy for hematologic cancers. ${ }^{14}$ All these various complications have involved high doses and lengthy periods of treatment. It cannot be inferred that these adverse effects have any relevance to the issue of corticosteroid use in the operating room where dexamethasone is given as a single small or moderate dose.

One potentially devastating adverse effect related to corticosteroids is osteonecrosis of the hip or other joint, which can have serious long-term effects on a patient's mobility. Mattano performed a retrospective review of 1,409 children who had received high-dose corticosteroid therapy for acute lymphoblastic leukemia and found that $111(7.9 \%)$ developed osteonecrosis. Of these children, $76.6 \%$ were ten to 15 years of age, and $3.6 \%$ were one to nine years of age. It should be emphasized that these patients received $235-470 \mathrm{mg} \cdot \mathrm{m}^{-2}$ of dexamethasone and/ or $3,600-7,000 \mathrm{mg} \cdot \mathrm{m}^{-2}$ of prednisone over a period of up to seven years. ${ }^{15}$ These results echo the findings of an older quantitative review of adult patients where it was found that there was a strong correlation of osteonecrosis with both high total dose and length of therapy with steroids. It was found, however, that bolus steroids or high-dose pulse therapy was not correlated with osteonecrosis. ${ }^{16}$ It seems, therefore, that osteonecrosis is associated with long-term steroid use, particularly if high doses are used, rather than with the single modest dose that is typically given in the operating room.

Another potential concern is that dexamethasone may destabilize blood glucose control in diabetics. While corticosteroids can increase blood glucose concentrations, the actual increase has not been shown to be significant in several studies. For example, Hans performed a prospective cohort study of 63 elective abdominal surgical patients (half of whom were type 2 diabetics) and administered 10 $\mathrm{mg}$ of dexamethasone perioperatively. ${ }^{17}$ Blood glucose in both diabetics and non-diabetics peaked at $120 \mathrm{~min}$ following dexamethasone. Nevertheless, the blood glucose concentration was only $1 \mathrm{mmol} \cdot \mathrm{L}^{-1}$ greater in the diabetic group than in the control. It could be argued that, while a slight increase in blood glucose might follow a dose of dexamethasone, the advantages of nausea control and an earlier return to a normal diet might well outweigh the risks in a diabetic patient. Although the data on corticosteroid use in diabetics primarily relate to adults, it can be assumed that the same considerations would apply to children.

In 2008, a paper was published by Czarnetzki et al. in the Journal of the American Medical Association that caused a flurry of concern about the risk of postoperative bleeding in pediatric tonsillectomy patients who had received dexamethasone. ${ }^{18}$ This arose from an investigation initially designed as a dose-finding study to determine the optimal amount of dexamethasone to prevent PONV. The study was terminated prematurely, as there appeared to be a higher than expected incidence of postoperative bleeding. The authors concluded that dexamethasone was associated with an increased risk of bleeding. This study was criticized for various methodological reasons. Many determinants of posttonsillectomy hemorrhage were not standardized; there was a higher than expected incidence of hemorrhage, particularly with some surgeons; ibuprofen was allowed to be given; the study was not powered to address hemorrhage; and some patients required a return to surgery on the day of the tonsillectomy, suggesting an issue with surgical technique. Two well-conducted meta-analyses have since failed to establish any relationship between post-tonsillectomy bleeding and the use of dexamethasone. ${ }^{19,20}$ Neither of these meta-analyses showed a relationship between steroid dose and postoperative bleeding. Yet another meta-analysis also showed no increase in bleeding events after tonsillectomy with dexamethasone. ${ }^{21}$ This meta-analysis, however, appeared to show a higher incidence of bleeding requiring a re-intervention if dexamethasone had been given. A closer look at the data, however, showed that many of these patients were in the flawed Czarnetzki study, and if this study had been excluded, there would have been no difference in this outcome. Additionally, Gallagher et al. have performed a randomized controlled non-inferiority trial in 314 pediatric tonsillectomy patients. The authors looked at postoperative bleeding as a primary end point in patients receiving either dexamethasone or placebo. ${ }^{22}$ They found that there was no increase in clinically significant bleeding, which was defined as bleeding that required readmission or reoperation. The evidence seems to be convincing, therefore, that dexamethasone does not cause clinically important post-tonsillectomy bleeding.

There have also been rare but extremely concerning case reports of patients developing tumour lysis syndrome (TLS) following perioperative use of dexamethasone. One case involved a 42-yr-old female who received dexamethasone during a biopsy procedure for suspected lymph node malignancy. ${ }^{23}$ The second case involved a developmentally delayed three-year-old male with Xq28 microdeletion. ${ }^{24} \mathrm{He}$ was known to have hypochromic microcytic anemia with splenomegaly, but he had no recent preoperative blood count before his adenotonsillectomy. On the sixth postoperative hour, the child suffered a cardiorespiratory arrest, and following resuscitation, he was found to have undiagnosed acute lymphoblastic leukemia and presumed dexamethasoneinduced TLS. He died three days postoperatively despite aggressive treatment, including extracorporeal membrane oxygenation. These two cases highlight the fact that perioperative dexamethasone should not be given in patients with known or possible hematologic malignancies who are at risk for TLS. Nevertheless, given the rarity of such reports and the widespread use of dexamethasone during anesthesia, this complication must be regarded as exceptionally uncommon. 
In the anesthesia literature, there is much evidence to support the notion of minimal harm from the administration of a single dose of dexamethasone. A recent large Cochrane review that included 1,756 pediatric tonsillectomy patients showed no harmful effects from a single perioperative dose of dexamethasone. ${ }^{7}$ In adults, De Oliveira performed a meta-analysis on 24 randomized controlled trials evaluating perioperative dexamethasone in 2,751 patients and found that there was significant improvement in pain but no increase in postoperative complications. ${ }^{25}$ A more recent meta-analysis of 45 studies involving 5,796 adults again showed the analgesic benefits of dexamethasone but no increase in rates of infection or delayed wound healing. ${ }^{26}$ When considering all this evidence, it is important to remember that, when events are rare, any statistical method used to analyze such events will have certain shortcomings. Bradburn points out that "many of the commonly used methods for meta-analysis give inappropriate answers when data are sparse." 27 Nevertheless, in the absence of long-term safety data from large clinical databases, these meta-analyses are the best currently available evidence.

Large single doses of dexamethasone have been used outside of the operating room for other clinical indications. Bjornson studied the efficacy of dexamethasone to treat croup in a randomized controlled trial of 720 children. ${ }^{28} \mathrm{~A}$ single $0.6 \mathrm{mg} \cdot \mathrm{kg}^{-1}$ dose of dexamethasone was given in the emergency department, and there were no reports of any adverse events on follow-up after 21 days. This dose is significantly greater than the dose typically used in the perioperative period.

Corticosteroids have been used extensively in the intensive care unit setting for the treatment of sepsis. In a large meta-analysis of mainly adult patients, the authors found that septic patients given short-term (one to three days) corticosteroids had no difference in 28-day all-cause mortality compared with controls. ${ }^{29}$ Most of the literature concerning corticosteroid use in sepsis relates to adult patients; however, in one randomized controlled trial, children with sepsis were administered a $0.20 \mathrm{mg} \cdot \mathrm{kg}^{-1}$ dose of dexamethasone eight hourly for two days, and results showed no difference in mortality. ${ }^{30}$ Seeing as there is no good evidence that the use of short-term high-dose steroids causes harm in septic patients, the chance of dexamethasone exacerbating any kind of infection in most surgical patients is unlikely.

In summary, a critical evaluation of the evidence accumulated over more than half a century would suggest that a single modest perioperative dose of dexamethasone in children is largely a safe intervention, with the possible exception of patients who have hematological malignancies. Other theoretical concerns do not seem to be a reason for withholding this efficacious antiemetic, anti-inflammatory, and co-analgesic drug. There is evidence, however, that a fairly small dose of dexamethasone may be all that is required to confer these benefits, ${ }^{31}$ so it would make sense, as with any drug, to use the lowest effective dose to achieve the required results.

\section{Innocuité de la dexaméthasone administrée en périopératoire chez l'enfant : est-il temps d'y repenser?}

La première utilisation thérapeutique de la cortisone rapportée chez l'humain a eu lieu en 1948. La patiente était une femme de 29 ans traitée à la Clinique Mayo en raison d'une polyarthrite rhumatoïde grave. Après 60 jours de traitement, ses symptômes rhumatoïdes s'étaient améliorés, mais le traitement a été interrompu en raison d'effets secondaires tels que l'hirsutisme, l'acné et l'idéation hostile. ${ }^{1}$ Il faut noter que ce premier essai de traitement thérapeutique aux corticostéroïdes a été interrompu en raison de complications liées au médicament. L'objectif de cet éditorial est de passer en revue les données probantes cliniques concernant le profil d'innocuité de la dexaméthasone, un corticostéroïde de synthèse, ce médicament étant communément utilisé en période périopératoire aujourd'hui, tant chez l'enfant que chez l'adulte. Des inquiétudes pourraient perdurer dans l'esprit de certains anesthésiologistes, craignant que les effets secondaires potentiels d'une dose unique et modeste de dexaméthasone soient importants. Existe-t-il des données probantes justifiant ces craintes? Les enfants courent-ils un risque plus ou moins élevé d'effets secondaires potentiels que les adultes? Est-ce que la dose et sa fréquence constituent une considération importante? Existe-t-il des données probantes suffisamment claires pour répondre à ces questions en 2013, ou d'autres recherches sont-elles nécessaires?

La dexaméthasone, un corticostéroïde de synthèse, est le résultat de recherches réalisées par la Schering Corporation et les Laboratoires Merck, publiées en 1958. ${ }^{2}$ Au cours des deux décennies suivantes, l'utilisation de la dexaméthasone en tant qu'agent anti-inflammatoire puissant a été explorée, et on a rapidement découvert que le médicament offrait des avantages dans des situations cliniques telles que l'extraction des dents de sagesse ${ }^{3}$ et la laryngotrachéobronchite aiguë (croup). ${ }^{4} \mathrm{Il}$ a fallu davantage de temps avant de reconnaître l'action antiémétique puissante de la dexaméthasone, bien que cet effet ait déjà été démontré dans les premières études. ${ }^{3}$ Dans les années 1980, la dexaméthasone a trouvé un rôle 
dans la prévention des vomissements induits par la chimiothérapie $;{ }^{5}$ il a toutefois fallu une autre décennie au moins avant que les implications d'une telle découverte pour le contrôle des nausées et vomissements postopératoires (NVPO) soient pleinement appréciées. La Society for Ambulatory Anesthesia a évalué de façon critique les données probantes concernant la prévention et la prise en charge adaptées des NVPO, et les directives qui se sont ensuivies recommandent l'utilisation de dexaméthasone en tant qu' antiémétique prophylactique efficace chez les enfants à la dose de $150 \mu \mathrm{g} \cdot \mathrm{kg}^{-1}$, jusqu'à un maximum de $5 \mathrm{mg}$. ${ }^{6}$ Les autres utilisations périopératoires de la dexaméthasone chez l'enfant se sont concentrées sur l'analgésie après des interventions telles que les amygdalectomies ${ }^{7}$ et les orchidopexies. ${ }^{8}$ Les divers avantages de la dexaméthasone sont si bien acceptés que ce médicament est aujourd'hui communément utilisé en période périopératoire, en général en dose unique modeste. Existe-t-il des données probantes suggérant que cette pratique comporte un risque significatif - particulièrement pour l'enfant?

La plupart des données probantes concernant les effets néfastes liés à l'utilisation de corticostéroïdes proviennent de la littérature ne portant pas spécifiquement sur l'anesthésie. Par exemple, les corticostéroïdes sont l'un des éléments principaux du traitement de l'asthme. Chez les enfants asthmatiques, une utilisation quotidienne de corticostéroïdes par voie orale ou inhalée a été associée à une diminution de la croissance. ${ }^{9}$ Les cliniciens ont également craint que les corticostéroïdes soient associés aux ulcères gastroduodénaux, mais les données probantes laissent à penser qu'il s'agit là davantage d'un mythe que de la réalité. ${ }^{10,11}$ Des effets secondaires psychologiques néfastes liés à l'utilisation de corticostéroïdes sont également possibles chez l'enfant, et ils peuvent prendre la forme d'un comportement agressif ou de trouble d'hyperactivité avec déficit de l'attention. Ces effets ont été démontrés dans des études évaluant des enfants subissant un traitement aux corticostéroïdes à dose élevée à long terme pour traiter une leucémie aiguë lymphoblastique et une affection abdominale inflammatoire. ${ }^{12,13}$ Des troubles du sommeil ont également été rapportés, mais il a été démontré que cet effet survenait principalement chez les patients recevant un traitement aux stéroïdes à dose élevée pour traiter un cancer hématologique. ${ }^{14}$ Toutes ces complications sont survenues suite à l'administration de doses élevées sur des périodes de traitement prolongées. On ne peut donc conclure que ces effets secondaires défavorables soient véritablement pertinents à la question de l'utilisation de corticostéroïdes en salle d'opération, là où la dexaméthasone n'est donnée qu'à une dose unique faible ou modérée.

Un effet secondaire potentiellement dévastateur lié aux corticostéroïdes est l'ostéonécrose de la hanche ou d'une autre articulation, qui peut avoir de graves effets sur la mobilité d'un patient à long terme. Mattano a publié un compte-rendu rétrospectif portant sur 1409 enfants ayant reçu un traitement aux corticostéroïdes à dose élevée pour traiter une leucémie aiguë lymphoblastique et a découvert que $111(7,9 \%)$ patients avaient développé une ostéonécrose. Parmi ces enfants, $76,6 \%$ étaient âgés de 10 à 15 ans, et 3,6\% avaient entre un et neuf ans. Il convient de souligner que ces patients ont reçu $235-470 \mathrm{mg} \cdot \mathrm{m}^{-2}$ de dexaméthasone et/ou $3600-7000 \mathrm{mg} \cdot \mathrm{m}^{-2}$ de prednisone sur une période pouvant aller jusqu'à sept ans. ${ }^{15}$ Ces résultats sont l'écho d'observations rapportées dans un compte-rendu quantitatif plus ancien qui s'intéressait à des patients adultes et dans lequel on a découvert une importante corrélation entre l'ostéonécrose et une dose totale élevée de corticostéroïdes d'une part et entre l'ostéonécrose et la durée du traitement d'autre part. On a toutefois découvert que les corticostéroïdes administrés en bolus et la thérapie pulsée à dose élevée n'étaient pas corrélés avec l'ostéonécrose. ${ }^{16}$ Par conséquent, il semble que l'ostéonécrose soit associée à l'utilisation à long terme de stéroïdes, particulièrement en cas de doses élevées, plutôt qu'à la dose unique modeste généralement administrée en salle d'opération.

Une autre crainte potentielle réside dans le fait que la dexaméthasone pourrait déséquilibrer le contrôle de la glycémie chez les diabétiques. Bien que les corticostéroïdes puissent augmenter les concentrations de glucose, plusieurs études n'ont pas réussi à démontrer que l'augmentation réelle était significative. Par exemple, Hans a réalisé une étude de cohorte prospective portant sur 63 patients devant subir une chirurgie abdominale non urgente (dont la moitié était constituée de diabétiques de type 2) et administré $10 \mathrm{mg}$ de dexaméthasone en période périopératoire ${ }^{17}$ La glycémie a atteint un pic à $120 \mathrm{~min}$ après l'administration de dexaméthasone, tant chez les diabétiques que chez les non-diabétiques. Toutefois, la glycémie n'était que de $1 \mathrm{mmol} \cdot \mathrm{L}^{-1}$ plus élevée dans le groupe diabétique que dans le groupe témoin. On pourrait soutenir que, malgré la survenue possible d'une légère augmentation de la glycémie après l'administration d'une dose de dexaméthasone, les avantages que cet agent offre en matière de contrôle des nausées et de retour plus rapide à un régime normal pourraient l'emporter sur les risques pour un patient diabétique. Bien que les données concernant l'utilisation de corticostéroïdes chez les diabétiques portent principalement sur des adultes, on peut conjecturer que les mêmes considérations s'appliqueraient aux enfants.

En 2008, Czarnetzki et coll. publiaient un article dans le Journal of the American Medical Association qui a suscité une recrudescence des inquiétudes concernant le risque de saignement postopératoire chez les enfants subissant une amygdalectomie et ayant reçu de la dexaméthasone. ${ }^{18}$ Ces inquiétudes sont apparues à l'issue d'un projet de recherche dont l'objectif initial était de rechercher la dose optimale de dexaméthasone à administrer afin de prévenir les NVPO. 
L'étude a été interrompue prématurément en raison de l'apparente incidence accrue de saignements postopératoires, plus abondants que prévu. Les auteurs ont conclu que la dexaméthasone était associée à un risque accru de saignement. Cette étude a été critiquée pour diverses raisons méthodologiques, notamment: plusieurs des facteurs déterminant l'hémorragie post-amygdalectomie n'étaient pas standardisés; l'incidence d'hémorragie était plus élevée que prévue, particulièrement avec certains chirurgiens; on a autorisé l'administration d'ibuprofène; l'étude n'avait pas la puissance pour traiter de la question de l'hémorragie; et certains patients ont dû retourner en chirurgie le jour de l'amygdalectomie, ce qui laisse à supposer que la technique chirurgicale posait problème. Depuis la parution de cette étude, deux excellentes méta-analyses ne sont pas parvenues à établir de lien entre les saignements post-amygdalectomie et l'utilisation de dexaméthasone. ${ }^{19,20} \mathrm{Ni}$ l'une ni l'autre de ces méta-analyses n'a montré de lien entre la dose de corticostéroïdes et les saignements postopératoires. Une autre méta-analyse n'a pas non plus montré d'augmentation des saignements après une amygdalectomie avec la dexaméthasone. $^{21}$ Toutefois, cette dernière méta-analyse a fait état d'une incidence plus élevée de saignements nécessitant une réintervention lors de l'administration de dexaméthasone. Néanmoins, en examinant les données de plus près, on remarque que bon nombre des patients en question étaient en fait ceux de l'étude de Czarnetzki, qui comporte des lacunes. Si cette étude avait été exclue, il n'y aurait pas eu de différence dans la méta-analyse à ce niveau-là. En outre, Gallagher et coll. ont réalisé une étude randomisée contrôlée de non-infériorité auprès de 314 patients pédiatriques devant subir une amygdalectomie. Le critère d'évaluation principal de cette étude était le saignement postopératoire chez les patients recevant de la dexaméthasone ou un placebo. ${ }^{22}$ Les auteurs n'ont observé aucune augmentation en matière de saignement significatif d'un point de vue clinique, défini comme un saignement nécessitant une réadmission ou une réopération. Par conséquent, les données probantes selon lesquelles la dexaméthasone ne cause pas de saignement important d'un point de vue clinique après une amygdalectomie semblent convaincantes.

Il existe également des présentations de cas, rares mais extrêmement inquiétantes, de patients manifestant un syndrome de lyse tumorale (SLT) après l'utilisation de dexaméthasone en période périopératoire. L'un des cas rapportés est celui d'une femme de 42 ans ayant reçu de la dexaméthasone pendant une biopsie pour dépister un possible ganglion lymphatique malin. ${ }^{23}$ Le second cas traitait d'un garçon de trois ans en retard de développement ayant une microdélétion du chromosome Xq28. ${ }^{24}$ On savait qu'il souffrait d'anémie microcytique hypochromique accompagnée d'une splénomégalie, mais aucune numération plaquettaire préopératoire récente n'avait été réalisée avant son adéno-amygdalectomie. Six heures après l'opération, l'enfant a subi un arrêt cardiorespiratoire, et après avoir été réanimé, on a découvert qu'il souffrait d'une leucémie aiguë lymphoblastique non diagnostiquée ainsi que d'un présumé SLT induit par la dexaméthasone. Il est mort trois jours après l'opération malgré un traitement énergique, y compris par oxygénation par membrane extracorporelle. Ces deux cas soulignent le fait que la dexaméthasone ne devrait pas être administrée en période périopératoire à des patients souffrant de malignités hématologiques connues ou suspectées et courant un risque de SLT. Ceci étant dit, étant donné la rareté de tels comptes rendus et l'utilisation courante de dexaméthasone pendant l'anesthésie, cette complication doit être considérée comme exceptionnellement rare.

Dans la littérature d'anesthésie, de nombreuses données probantes soutiennent la notion que l'administration d'une dose unique de dexaméthasone ne fait qu'un tort minime. Un compte rendu récent portant sur la base de données Cochrane a inclus 1756 enfants ayant subi une amygdalectomie et n'a montré aucun effet néfaste à la suite d'une dose périopératoire unique de dexaméthasone. ${ }^{7} \mathrm{~S}$ 'intéressant à une population adulte, De Oliveira a réalisé une méta-analyse de 24 études randomisées contrôlées évaluant l'utilisation périopératoire de dexaméthasone chez 2751 patients; il n'a pas observé d'amélioration significative de la douleur, mais pas non plus d'augmentation des complications postopératoires. ${ }^{25}$ Une méta-analyse plus récente examinant 45 études incluant un total de 5796 adultes a elle aussi démontré les avantages de la dexaméthasone comme analgésique, sans pour autant observer d'augmentation du taux d'infection ou de retard dans la guérison des plaies. ${ }^{26}$ Lorsqu'on évalue toutes ces données probantes, il importe de se souvenir que, lorsqu'un événement est rare, toute méthode statistique utilisée pour l'analyser comportera forcément certaines lacunes. Bradburn fait remarquer que «bon nombre des méthodes communément utilisées dans les méta-analyses donnent des réponses inadaptées lorsque les données sont rares. ${ }^{27}$ Toutefois, en l'absence de données d'innocuité à long terme tirées de bases de données cliniques d'envergure, ces méta-analyses sont les meilleures données probantes dont nous disposions à l'heure actuelle.

D'importantes doses uniques de dexaméthasone ont été utilisées hors de la salle d'opération pour d'autres indications cliniques. Bjornson a étudié l'efficacité de la dexaméthasone pour traiter le croup dans une étude randomisée contrôlée portant sur 720 enfants. ${ }^{28}$ Une dose unique de $0,6 \mathrm{mg} \cdot \mathrm{kg}^{-1}$ de dexaméthasone a été donnée à l'urgence, et aucun événement néfaste n'a été rapporté lors du rendez-vous de suivi, 21 jours plus tard. Cette dose est considérablement plus élevée que la dose généralement administrée en période périopératoire. 
On a beaucoup utilisé les corticostéroïdes pour traiter le sepsis à l'unité des soins intensifs. Dans une importante méta-analyse incluant principalement des patients adultes, les auteurs ont découvert que les patients septiques à qui l'on avait donné des corticostéroïdes à court terme (un à trois jours) ne montraient aucune différence en matière de mortalité à 28 jours, toutes causes confondues, par rapport aux témoins. ${ }^{29}$ La plupart des études abordant la question de l'utilisation de corticostérö̈des pour traiter le sepsis portent sur des patients adultes; toutefois, dans une étude randomisée contrôlée, on a administré une dose de 0,20 $\mathrm{mg} \cdot \mathrm{kg}^{-1}$ de dexaméthasone à des enfants atteints de sepsis toutes les huit heures pendant deux jours, et les résultats n'ont pas montré de différence en matière de mortalité. ${ }^{30}$ Comme il n'existe pas de données probantes convaincantes selon lesquelles l'utilisation à court terme de doses élevées de corticostéroïdes serait néfaste pour les patients septiques, il est peu probable que la dexaméthasone aggrave une infection, quelle qu'elle soit, chez la plupart des patients en chirurgie.

Pour résumer, une évaluation critique des données probantes accumulées au cours de plus de 50 ans laisse à penser qu'une dose unique et modeste de dexaméthasone, administrée à l'enfant en période périopératoire, est généralement sécuritaire - à l'exception possible des patients présentant des malignités hématologiques. Il ne semble pas que les autres inquiétudes théoriques justifient de ne pas administrer ce médicament antiémétique, anti-inflammatoire et co-analgésique efficace. Les données probantes suggèrent cependant qu'une dose relativement faible de dexaméthasone suffirait pour conférer ces avantages. ${ }^{31}$ Il serait donc logique, comme pour tout médicament, d'utiliser la dose efficace la plus faible pour atteindre les résultats souhaités.

Financial support No financial support was required for this study.

Conflicts of interest None declared.

Soutien financier Aucun soutien financier n'a été nécessaire pour cette étude.

Conflit d'intérêt Aucun

\section{References}

1. Benedek TG. History of the development of corticosteroid therapy. Clin Exp Rheumatol 2011; 29 (Suppl 68): S-5-12.

2. Bunim JJ, Black RL, Lutwak L, Peterson RE, Whedon DG. Studies on dexamethasone, a new synthetic steroid in rheumatoid arthritis - a preliminary report. Arthritis Rheum 1958; 1: 313-31.

3. Baxendale BR, Vater $M$, Lavery $K M$. Dexamethasone reduces pain and swelling following extraction of third molar teeth. Anaesthesia 1993; 48: 961-4.
4. Skolnik NS. Treatment of croup. Am J Dis Child 1989; 143: 1045-9.

5. Bakowski MT. Advances in anti-emetic therapy. Cancer Treat Rev 1984; 11: 237-56.

6. Gan TJ, Meyer TA, Apfel CC, et al. Society for Ambulatory Anesthesia guidelines for the management of postoperative nausea and vomiting. Anesth Analg 2007; 105: 1615-28.

7. Steward DL, Grisel J, Meinzen-Derr J. Steroids for improving recovery following tonsillectomy in children. Cochrane Database Syst Rev 2011; 8: CD003997.

8. Hong JY, Han SW, Kim WO, Kim EJ, Kil HK. Effect of dexamethasone in combination with caudal analgesia on postoperative pain control in day-case paediatric orchiopexy. Br J Anaesth 2010; 105: 506-10.

9. Allen DB, Mullen M, Mullen B. A meta-analysis of the effect of oral and inhaled corticosteroids on growth. J Allergy Clin Immunol 1994; 93: 967-76.

10. Conn HO, Poynard T. Corticosteroids and peptic ulcer: metaanalysis of adverse events during steroid therapy. J Intern Med 1994; 236: 619-32.

11. Martinek J, Hlavova K, Zavada F, et al. "A surviving myth" corticosteroids are still considered ulcerogenic by a majority of physicians. Scand J Gastroenterol 2010; 45: 1156-61.

12. Pound CM, Clark C, Ni A, Athale U, Lewis V, Halton JM. Corticosteroids, behavior, and quality of life in children treated for acute lymphoblastic leukemia; a multicentered trial. J Pediatr Hematol Oncol 2012; 34: 517-23.

13. Mrakotsky C, Forbes PW, Bernstein JH, et al. Acute cognitive and behavioral effects of systemic corticosteroids in children treated for inflammatory bowel disease. J Int Neuropsychol Soc 2013; 19: 96-109.

14. Hinds PS, Hockenberry MJ, Gattuso JS, et al. Dexamethasone alters sleep and fatigue in pediatric patients with acute lymphoblastic leukemia. Cancer 2007; 110: 2321-30.

15. Mattano LA Jr, Sather HN, Trigg ME, Nachman JB. Osteonecrosis as a complication of treating acute lymphoblastic leukemia in children: a report from the Children's Cancer Group. J Clin Oncol 2000; 18: 3262-72.

16. Felson DT, Anderson JJ. Across-study evaluation of association between steroid dose and bolus steroids and avascular necrosis of bone. Lancet 1987; 1: 902-6.

17. Hans $P$, Vanthuyne A, Dewandre PY, Brichant JF, Bonhomme V. Blood glucose concentration profile after $10 \mathrm{mg}$ dexamethasone in non-diabetic and type 2 diabetic patients undergoing abdominal surgery. Br J Anaesth 2006; 97: 164-70.

18. Czarnetzki C, Elia N, Lysakowski $C$, et al. Dexamethasone and risk of nausea and vomiting and postoperative bleeding after tonsillectomy in children: a randomized trial. JAMA 2008; 300: 2621-30.

19. Geva A, Brigger MT. Dexamethasone and tonsillectomy bleeding: a meta-analysis. Otolaryngol Head Neck Surg 2011; 144: 838-43.

20. Shargorodsky J, Hartnick CJ, Lee GS. Dexamethasone and postoperative bleeding after tonsillectomy and adenotonsillectomy in children: a meta-analysis of prospective studies. Laryngoscope 2012; 122: 1158-64.

21. Plante J, Turgeon AF, Zarychanski R, et al. Effect of systemic steroids on post-tonsillectomy bleeding and reinterventions: systematic review and meta-analysis of randomised controlled trials. BMJ 2012; 345: e5389.

22. Gallagher TQ, Hill C, Ojha S, et al. Perioperative dexamethasone administration and risk of bleeding following tonsillectomy in children: a randomized controlled trial. JAMA 2012; 308: 1221-6.

23. Chanimov M, Koren-Michowitz M, Cohen ML, Pilipodi S, Bahar $M$. Tumor lysis syndrome induced by dexamethasone. Anesthesiology 2006; 105: 633-4. 
24. McDonnell C, Barlow R, Campisi P, Grant R, Malkin D. Fatal peri-operative acute tumour lysis syndrome precipitated by dexamethasone. Anaesthesia 2008; 63: 652-5.

25. De Oliveira GS, Jr Almeida MD, Benzon HT, McCarthy RJ. Perioperative single dose systemic dexamethasone for postoperative pain: a meta-analysis of randomized controlled trials. Anesthesiology 2011; 115: 575-88.

26. Waldron NH, Jones CA, Gan TJ, Allen TK, Habib AS. Impact of perioperative dexamethasone on postoperative analgesia and side-effects: systematic review and meta-analysis. Br J Anaesth 2013; 110: 191-200.

27. Bradburn MJ, Deeks JJ, Berlin JA, Russell Localio A. Much ado about nothing: a comparison of the performance of metaanalytical methods with rare events. Stat Med 2007; 26: 53-77.

28. Bjornson CL, Klassen TP, Williamson J, et al. A randomized trial of a single dose of oral dexamethasone for mild croup. N Engl J Med 2004; 351: 1306-13.
29. Annane D, Bellissant E, Bollaert PE, Briegel J, Keh D, Kupfer Y. Corticosteroids for severe sepsis and septic shock: a systematic review and meta-analysis. BMJ 2004; 329: 480.

30. Slusher T, Gbadero D, Howard C, et al. Randomized, placebocontrolled, double blinded trial of dexamethasone in African children with sepsis. Pediatr Infect Dis J 1996; 15: 579-83.

31. Kim MS, Cote CJ, Cristoloveanu $C$, et al. There is no doseescalation response to dexamethasone $(0.0625-1.0 \mathrm{mg} / \mathrm{kg})$ in pediatric tonsillectomy or adenotonsillectomy patients for preventing vomiting, reducing pain, shortening time to first liquid intake, or the incidence of voice change. Anesth Analg 2007; 104: 1052-8. 